\title{
Controlling frontal photopolymerization with optical attenuation and mass diffusion
}

\author{
Matthew G. Hennessy, Alessandra Vitale, Omar K. Matar, and João T. Cabral* \\ Department of Chemical Engineering, Imperial College London, South Kensington Campus, London SW7 2AZ, United Kingdom
}

(Received 3 March 2015; revised manuscript received 17 April 2015; published 11 June 2015)

\begin{abstract}
Frontal photopolymerization (FPP) is a versatile directional solidification process that can be used to rapidly fabricate polymer network materials by selectively exposing a photosensitive monomer bath to light. A characteristic feature of FPP is that the monomer-to-polymer conversion profiles take on the form of traveling waves that propagate into the unpolymerized bulk from the illuminated surface. Practical implementations of FPP require detailed knowledge about the conversion profile and speed of these traveling waves. The purpose of this theoretical study is to (i) determine the conditions under which FPP occurs and (ii) explore how optical attenuation and mass transport can be used to finely tune the conversion profile and propagation kinetics. Our findings quantify the strong optical attenuation and slow mass transport relative to the rate of polymerization required for FPP. The shape of the traveling wave is primarily controlled by the magnitude of the optical attenuation coefficients of the neat and polymerized material. Unexpectedly, we find that mass diffusion can increase the net extent of polymerization and accelerate the growth of the solid network. The theoretical predictions are found to be in excellent agreement with experimental data acquired for representative systems.
\end{abstract}

DOI: 10.1103/PhysRevE.91.062402

PACS number(s): 68.08.-p, 82.35.-x, 81.30.Fb, 82.50.Hp

\section{INTRODUCTION}

Photopolymerization is a common solidification process that is used to create cross-linked polymer networks by subjecting a photosensitive monomer-rich bath to light, typically ultraviolet (uv) radiation [Fig. 1(a)]. The high polymerization rates that are achieved under intense radiation enable the rapid fabrication of complex patterned structures controlled through selective illumination of the bath, and development (or selected removal) of the unpolymerized material. Furthermore, it is possible to carry out photopolymerization at ambient temperatures and in open atmospheres, making this process particularly attractive from an industrial point of view [1-3].

The fundamental idea behind photopolymerization is that the absorption of radiation by photoinitiators within the monomer-rich bath initiates the necessary chemical reactions leading to polymerization and, often, simultaneous crosslinking. Light absorption causes the intensity of radiation to decay within the material, away from the illuminated surface [Fig. 1(b)]. Consequently, the monomer-to-polymer conversion is also spatially nonuniform, exhibiting a gradient orthogonal to the surface. Under certain experimental conditions that will be discussed below, photopolymerization can be confined to a localized region, a planar front, that propagates into the unpolymerized bulk along the illuminated direction. The corresponding conversion profile of the traveling wave, normal to the surface, can be represented by an order parameter $\phi$ measuring the local fraction of polymer [Fig. 1(c)]. The polymerization front can be formally defined as the position where the order parameter $\phi$ reaches a critical value of $\phi_{c}$, a characteristic of the polymer network. The front position and

\footnotetext{
*j.cabral@imperial.ac.uk
}

Published by the American Physical Society under the terms of the Creative Commons Attribution 3.0 License. Further distribution of this work must maintain attribution to the author(s) and the published article's title, journal citation, and DOI. its dependence on light exposure and material properties are particularly relevant for practical applications, as these provide a measure of the depth (or "height" following development) of the growing polymer network [Fig. 1(d)]. The appearance of a sharp wavefront separating polymer-rich (solid) and monomer-rich (liquid) phases is thus characteristic of a frontal photopolymerization (FPP) process [4-6]. The overall FPP physical scenario is, however, common to various directional solidification and degradation processes.

In FPP, the propagation of the wavefront is driven purely by the continuous absorption of radiation, making it distinct from thermal (TFP) and isothermal (IFP) frontal polymerization. Wave propagation in TPF is sustained by the heat released in exothermic chemical reactions and the Arrhenius-like dependence of the reaction rates. IFP occurs as a result of the Trommsdorff-Norrish effect, whereby polymerization leads to a local increase in the liquid viscosity, which, in turn, inhibits terminating reactions. TFP and IFP can be initiated through a localized heat source or a polymer seed, respectively, whereas FPP can begin as soon as the bath is illuminated with a sufficient radiation dose. A comprehensive and accessible review of these frontal polymerization processes is given by Pojman [7].

In practice, FPP is employed in the fields of lithography [4], rapid prototyping [8], coatings [9], dentistry [10], and biomedicine [11], where it has been used to fabricate microcircuits [2], microfluidic devices [12], and more recently, gradient polymer materials with tunable properties that vary in the direction of wave propagation as well as in the lateral plane orthogonal to it [13]. All of these examples demonstrate the exciting possibility of utilizing FPP to rapidly manufacture with light. Harnessing the full potential of FPP, however, requires a quantitative understanding of the travelingwave conversion profiles and how they propagate throughout the bulk. Elucidating the primary mechanisms underpinning photopolymerization processes has been the focus of several theoretical and experimental studies [5,6,14-18]. We have postulated that FPP should occur under conditions of strong optical attenuation and limited mass and thermal transport 


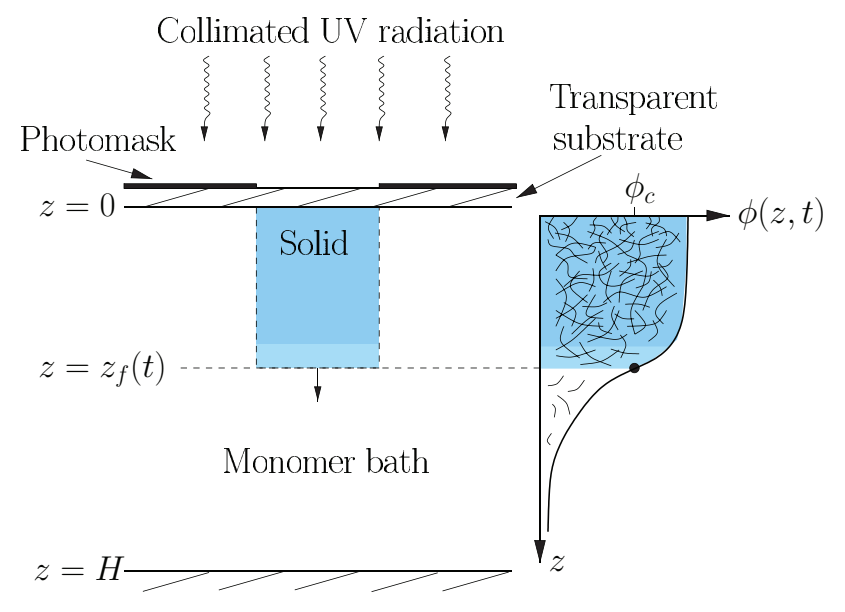

(a)
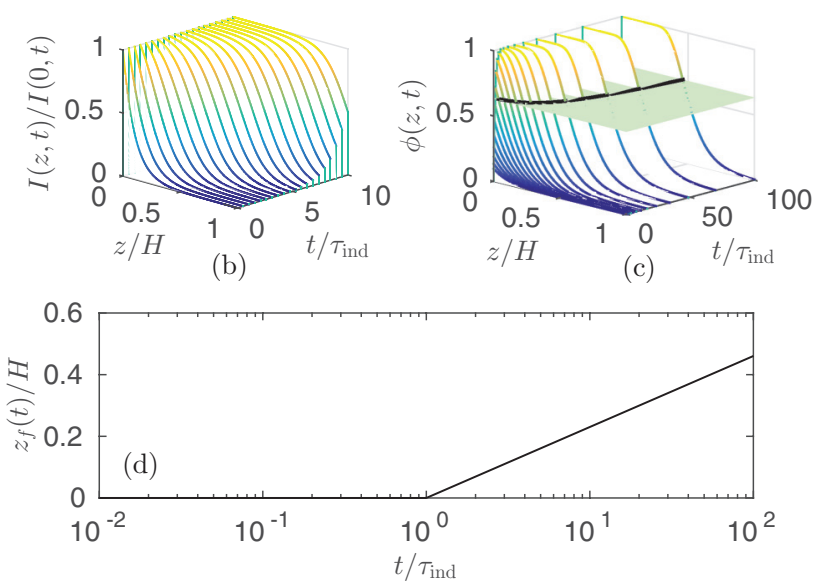

FIG. 1. (Color online) (a) Typical FPP process consisting of a photosensitive monomer-rich bath selectively illuminated by collimated uv radiation. The conversion profile can be described by an order parameter $\phi(z, t)$ representing the fraction of polymer at a point $z$ at time $t$. A solid network forms above a certain critical $\phi_{c}$ indicated by a solid circle. (b) The intensity of the radiation $I(z, t)$ decays as its passes through the bath, giving rise to a solid polymer network that grows from the illuminated surface into the bulk. (c) The spatiotemporal evolution of $\phi(z, t)$ shows the emergence of a traveling wave, a characteristic feature of FPP. The position of the wavefront, $z_{f}(t)$, corresponds to the location of the solid-liquid interface, formally defined by the position where $\phi$ reaches a prescribed value of $\phi_{c}$ (shown as the horizontal plane), characteristic of the network. (d) By tracking the temporal evolution of $z_{f}$, the depth (or "thickness") of the solidified section is obtained at each point in time. The solid network does not form instantly upon illumination, but only after an induction time $\tau_{\text {ind }}$ is exceeded, corresponding to the time required to first reach $\phi_{c}$.

$[4,5,13]$. However, to the best of our knowledge, there has been no systematic investigation into how these factors influence the appearance and/or the profile of traveling waves. The purpose of this paper is, therefore, to conduct a theoretical study of the photopolymerization process in order to (i) identify the conditions under which FPP occurs, and (ii) explore how optical attenuation and mass transport can alter the shape of the traveling wave. FPP experiments on model systems of varying attenuation and mass transfer are formulated and compared against model predictions. Particular attention will be paid to determining the key physical parameters that are responsible for selecting the width of the interfacial profile separating the polymer-rich $(\phi \simeq 1)$ and monomer-rich $(\phi \simeq 0)$ phases, depicted in Fig. 1(c). The material properties of the cured solid, e.g., the elastic modulus [13], can vary significantly over this layer; thus, the ability to control its thickness will be directly relevant to the fabrication of gradient polymer materials.

Previous theoretical studies of photopolymerization have led to the development of a broad range of mathematical models for this process. At one end of the spectrum are accurate physicochemical models accounting for each of the reaction steps (minimally photoinitiation, propagation, and termination) [19-22], nonuniform distributions of polymer chain length [23,24], generation and diffusion of thermal energy [15], mass transport [25], and intricate optical effects [26,27]. Such models, however, often suffer from an excessive number of parameters, some of which cannot be measured experimentally, and thus they offer limited insight and practical use. At the other end are reduced, coarse-grained, or minimal models that are largely phenomenological in nature, but which capture the spatiotemporal evolution of key quantities (e.g., patterned depth and light transmission) $[4,6,14]$. Minimal models are generally based on physically observable quantities and benefit from greater simplicity, thus making them amenable to mathematical analysis and FPP fabrication. For these reasons, we choose to extend the minimal model proposed by Cabral et al. [4], which has been shown to agree exceptionally well with experimental data for thiol-ene systems with low $\phi_{c}[5,13]$.

The outline of this paper is as follows. In Sec. II, we revisit our previous minimal FPP model [4] to gain direct insight into the mechanisms that control the shape of the traveling wave profile. We then incorporate mass diffusion into this model in Sec. III. Our theoretical results are compared with new and previously reported experiments in Sec. IV. We discuss our findings in a practical context and conclude the paper in Sec. V.

\section{NEW INSIGHTS FROM A "MINIMAL" FPP MODEL}

We begin our investigation by revisiting a one-dimensional (1D) mean-field model of photopolymerization that was introduced by Cabral et al. [4]. This model consists of two coupled nonlinear partial differential equations for (i) an order parameter $\phi=\phi(z, t)$ measuring the local extent of polymerization, and (ii) the intensity of radiation throughout the mixture $I=I(z, t)$, at a depth $z$ in the mixture at time $t$. The order parameter is referred to as the conversion fraction, and it satisfies $0 \leqslant \phi \leqslant 1$, with $\phi=0$ and 1 denoting regions of the mixture that have undergone no or complete polymerization (or cross-linking), respectively. In this regard, $\phi$ can be loosely interpreted as the polymerized fraction occupying the mixture, with $(1-\phi)$ corresponding to the fraction of unpolymerized monomer-rich liquid. Solidification occurs when $\phi$ reaches a certain critical $\phi_{c}$, and whose value depends on the reactive mixture functionality and architecture of the network and is thus system-specific. 
The model follows the experimental configuration depicted in Fig. 1(a); the monomer-rich bath is covered with a transparent substrate and illuminated from above. The bath is assumed to be of thickness $H$ so that the downward-pointing vertical coordinate $z$ is bounded between $0 \leqslant z \leqslant H$, with $z=0$ and $z=H$ denoting the top and bottom of the mixture, respectively. In this section, we shall assume that $H \rightarrow \infty$, which is common for multilevel lithographic patterning [4]. The intensity of incoming radiation at the top surface of the photosensitive material is denoted as $I_{0}$, and, therefore, $I(0, t)=I_{0}$ at all times.

In our original model, photopolymerization was taken to be isothermal, and mass transport was not considered; the latter will be introduced into the model in Sec. III. The reaction rate is assumed to be proportional to an effective rate coefficient $K$ with units of $\mathrm{m}^{2} \mathrm{~J}^{-1}$, the fraction of available sites $(1-\phi)$, and the intensity of radiation, $I$. The conversion fraction $\phi$, therefore, satisfies

$$
\frac{\partial \phi}{\partial t}=K(1-\phi) I .
$$

As the radiation passes through the mixture, it is absorbed by the constituent chemical components; indeed, it is this absorption that leads to photoinitiation and then polymerization. Due to this absorption, the intensity of radiation decays as the distance from the illuminated surface increases. This is modeled through a generalized Beer-Lambert law given by

$$
\frac{\partial I}{\partial z}=-\bar{\mu}(\phi) I
$$

where $\bar{\mu}$ is the optical attenuation coefficient of the mixture. The monomer-rich and polymer-rich phases are generally expected to have different absorption coefficients given by $\mu_{0}$ and $\mu_{\infty}$, respectively; therefore, the absorption coefficient of the mixture is based on a phase average of the two:

$$
\bar{\mu}(\phi)=\mu_{0}(1-\phi)+\mu_{\infty} \phi,
$$

which varies with $z$ and $t$ during the process. The model is closed by imposing an initial condition for the conversion fraction and a boundary condition for the intensity. Trivially, we take the initial mixture to be unpolymerized, and the intensity of radiation at the surface, $I_{0}$, to be fixed. Therefore, we impose the conditions

$$
\begin{aligned}
& \phi(z, t=0)=0, \quad \forall z, \\
& I(z=0, t)=I_{0}, \quad \forall t .
\end{aligned}
$$

The solutions of this model have been previously examined, and it has been noted that the conversion fraction $\phi$ takes the form of a traveling wave after a sufficiently large time interval has passed [5]. An example of such a wave is shown in Fig. 1(c); regions far ahead of and behind the wave correspond to unpolymerized liquid $(\phi=0)$ and fully polymerized solid $(\phi=1)$, respectively. Warren et al.[6] have shown that the profile of this traveling wave can be obtained by solving the equation

$$
\frac{\partial \hat{\phi}}{\partial \hat{z}}=-(1-\hat{\phi})\left[\left(\mu_{0}-\mu_{\infty}\right) \hat{\phi}-\mu_{\infty} \log (1-\hat{\phi})\right],
$$

where $\hat{z}=z-z_{f}(t)$ measures distances relative to the front of the wave $z_{f}(t)$, and $\hat{\phi}(\hat{z})=\phi(z, t)$. Carets are used to denote quantities in the coordinate frame of the moving front. The far-field boundary condition given by $\hat{\phi}(\hat{z} \rightarrow \infty) \rightarrow 0$ is automatically satisfied by solutions to this equation; therefore, an additional boundary condition is required to uniquely determine the solution. The need to impose an extra boundary condition is related to the various possible definitions for the wavefront $z_{f}(t)$. In physical terms, this wavefront corresponds unambiguously to the solid-liquid interface, which is obtained following pattern development (i.e., selective dissolution of the liquid phase). One possibility is thus to define the interface by a critical value of the conversion fraction, $\phi_{c}$, which can be interpreted as a gelation or percolation point. This $\phi_{c}$ can effectively be obtained experimentally and is an intrinsic property of the network-forming system $[4,5,13]$. In this case, the additional boundary condition reads $\hat{\phi}(0)=\phi_{c}$. Alternatively, the position of the interface can be defined as the inflection point of $\hat{\phi}$, in which case the additional condition is given by $\hat{\phi}^{\prime \prime}(0)=0$, where the prime denotes differentiation with respect to $\hat{z}$. Mathematically, these formulations are equivalent and they simply reflect different choices for the origin.

The wavefront position $z_{f}(t)$ can be obtained by first noticing that (1a) can be solved analytically at the point $z=0$ using $I(0, t)=I_{0}$ to give $\phi(0, t)=1-\exp \left(-K I_{0} t\right)$. After fixing the origin and obtaining the unique solution of (2), the front propagation kinetics can be found via the condition

$$
\hat{\phi}\left[-z_{f}(t)\right]=\phi(0, t)=1-\exp \left(-K I_{0} t\right) .
$$

We note here that the functional form of $z_{f}$ will depend on the precise condition defining the solid-liquid interface.

Using this model, it is possible to determine the experimental conditions that control the width of the diffuse solidliquid interface. This is practically important as development truncates the front profile yielding a sharp planar interface, albeit with a gradient network structure within the solid phase. There is some flexibility in defining this interfacial width, and we adopt the convention that the interface is located at the inflection point of $\phi$, corresponding to $\hat{\phi}^{\prime \prime}(0)=0$. The width of the interface, $w$, is defined as the inverse of the derivative at this point, i.e.,

$$
w \equiv \frac{1}{\left|\hat{\phi}^{\prime}(0)\right|} .
$$

As shown in Fig. 2, this definition is exact when $\hat{\phi}(0)=1 / 2$ and when the conversion profile is symmetric about the inflection point. In general, however, the conversion profiles are not symmetric; therefore, the expression in (4) only provides an estimate of the width rather than its true value. The conditions under which this estimate is accurate will be specified below. Although we have previously reported [6] this form for the interfacial width, we now examine its implications in detail.

From (2), it follows that the conversion profile $\hat{\phi}$ can only be changed through the optical attenuation coefficients, $\mu_{0}$ and $\mu_{\infty}$; therefore, it is these two parameters that influence $w$ and hence control the sharpness of the solidification front profile. To evaluate this dependence, we first consider two 


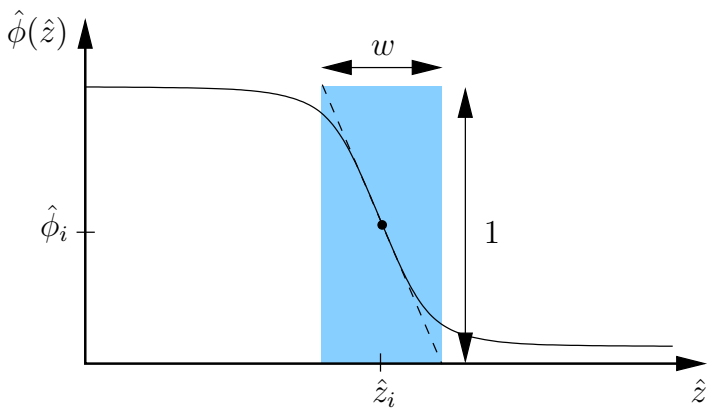

FIG. 2. (Color online) Schematic diagram showing the width, $w$, of the diffuse interfacial layer separating polymer-rich and monomer-rich phases. If the conversion profile $\hat{\phi}$ is symmetric about its inflection point located at $\hat{z}_{i}$ and satisfies $\hat{\phi}\left(\hat{z}_{i}\right) \equiv \hat{\phi}_{i}=1 / 2$, then simple trigonometry yields $w=1 /\left|\hat{\phi}^{\prime}\left(\hat{z}_{i}\right)\right|$. For asymmetric conversion profiles, this expression for $w$ becomes approximate with an accuracy depending on the degree of asymmetry.

types of photopolymerization for which the model has simple closed-form solutions. In the case of photoinvariant FPP, the optical attenuation of the mixture effectively remains constant over time; that is, the optical properties of the solid and liquid phases are equal: $\mu_{0}=\mu_{\infty}=\bar{\mu}$. Equations (2) and (3) are readily solved to yield

$$
\begin{aligned}
\hat{\phi}(\hat{z}) & =1-\exp \left(-e^{-\bar{\mu} \hat{z}}\right), \\
z_{f}(t) & =\bar{\mu}^{-1} \log \left(K I_{0} t\right), \\
w & =e^{1} \bar{\mu}^{-1} .
\end{aligned}
$$

Since the front position $z_{f}$ must be positive, we obtain $t>\left(K I_{0}\right)^{-1}$. The factor $\tau_{\text {ind }} \equiv\left(K I_{0}\right)^{-1}$ corresponds thus to an induction time, reflecting the finite light exposure time required to first reach the solidification condition, since an interface does not instantaneously form when the system is irradiated. Had a different criterion for the position of the interface been used, the corresponding induction time would change slightly. For instance, if the interface was defined by a critical value of the conversion profile, $\phi_{c}$, then the induction time would become

$$
t_{\text {ind }}=\tau_{\text {ind }} \log \left(\frac{1}{1-\phi_{c}}\right)
$$

By setting $\phi_{c}$ equal to the value of $\hat{\phi}$ at the inflection point, $\hat{\phi}(0) \equiv \hat{\phi}_{i}=1-e^{-1}$, the original induction time can be recovered, i.e., $t_{\text {ind }}=\tau_{\text {ind }}$, thus demonstrating the interchangeability of these two definitions of the interface. Experimentally, we find that an empirical $\phi_{c}$ is required to track the measured front kinetics of a range of neat [4] and nanofilled [5] thiol-ene systems.

An alternative photopolymerization situation to consider is strong photobleaching, which is expected upon activation of the photoinitiator [3], eventually resulting in a solid phase that is much less absorbing than the starting liquid: $\mu_{\infty} \ll \mu_{0}$. By making the approximation $\mu_{\infty} / \mu_{0} \rightarrow 0$, we find that the solution and the width are given by

$$
\begin{aligned}
\hat{\phi}(\hat{z}) & =\frac{1}{1+\exp \left(\mu_{0} \hat{z}\right)}, \\
z_{f}(t) & =\mu_{0}^{-1}\left[K I_{0} t+\log \left(1-e^{-K I_{0} t}\right)\right], \\
w & =4 \mu_{0}^{-1} .
\end{aligned}
$$

In this case, the induction time is given by $\tau_{\text {ind }} \log 2$, with $\tau_{\text {ind }}$ defined as before. By dimensional considerations, we can conclude that the induction time will always be the product of a dimensionless numerical factor and $\tau_{\text {ind }}$, regardless of the relative sizes of the attenuation coefficients.

For other combinations of the attenuation coefficients, the solution for the conversion fraction can only be written in terms of nonelementary functions, as discussed by Warren et al.[6]. Despite this fact, an analytical estimate for the width of the interface can remarkably still be obtained if the condition in (4) is used. The value of $\hat{\phi}$ at its inflection point can be determined by differentiating (2) with respect to $\hat{z}$, substituting (2) into this expression, and solving the equation to find

$$
\hat{\phi}_{i}=1-\frac{1}{2} \frac{\mathrm{W}\left[2\left(\mu_{r}-1\right) e^{\mu_{r}-2}\right]}{\mu_{r}-1},
$$

where $\mathrm{W}$ is the Lambert $\mathrm{W}$ function and $\mu_{r}=\mu_{0} / \mu_{\infty}$. The width of the interface is then given by

$$
w=\frac{4\left(\mu_{r}-1\right) \mu_{\infty}^{-1}}{\mathrm{~W}\left[2\left(\mu_{r}-1\right) e^{\mu_{r}-2}\right]\left\{\mathrm{W}\left[2\left(\mu_{r}-1\right) e^{\mu_{r}-2}\right]+2\right\}} .
$$

This expression can be used to estimate the interfacial width in the limit of strong photodarkening, which occurs when the solid absorbs much more radiation than the liquid, $\mu_{0} \ll \mu_{\infty}$. In this case, we find that

$$
w \simeq 6.18 \mu_{\infty}^{-1} .
$$

A contour plot of the function $w$ in (4) can be used to examine how the width varies with both of the attenuation coefficients, which is shown in Fig. 3(a). It can be seen that the width of the interface is substantially decreased in strongly absorbing media, and increases without bound as both attenuation coefficients decrease to zero. The latter corresponds to the situation in which the intensity of radiation remains approximately constant throughout the material, leading to nearly uniform polymerization rather than a traveling polymerization wave propagating from the illuminated surface.

To assess how well the expression in (9) characterises the interfacial width, we examine the value of the conversion fraction at the interface using (8) as well as the conversion profiles themselves in the three main photopolymerization cases described above. The dependence of $\hat{\phi}_{i}$ on the ratio of attenuation coefficients, $\mu_{r}$, is shown in Fig. 3(b). The curve decreases monotonically from its value of $\hat{\phi}_{i} \simeq 0.8$ when $\mu_{r}=0$ and it asymptotes to a value of $1 / 2$ as $\mu_{r} \rightarrow \infty$. The large deviation of $\hat{\phi}_{i}$ from the ideal value of $1 / 2$ that occurs for $\mu_{r} \ll 1$ is indicative that $w$ may be a poor predictor of the interfacial width in this regime. Indeed, a visual inspection of the conversion profile in the case of strong photodarkening ( $\mu_{r} \simeq 0$ ), which is plotted in Fig. 4(a), shows that the width of the interfacial layer is much larger than the prediction of $6.18 \mu_{\infty}^{-1}$ given in (10). In this case, the error in $w$ is due 


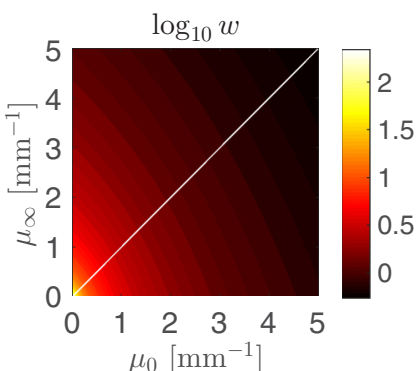

(a)

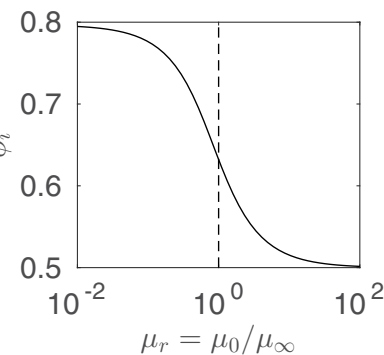

(b)
FIG. 3. (Color online) (a) The functional dependence of the width of the diffuse solid-liquid interface on the attenuation coefficients in the solid and liquid phases, $\mu_{\infty}$ and $\mu_{0}$, respectively. Here $w$ is computed from (9), and it will have units of mm. The white line denotes the curve $\mu_{\infty}=\mu_{0}$. In the region below this line, the conversion profiles are expected to be relatively symmetric about the interface. (b) The dependence of the conversion fraction at its inflection point as a function of the ratio of attenuation coefficients $\mu_{r}$. This curve has been computed from (8) and it can be used to assess the symmetry of the conversion profile and the accuracy of the width estimate; see text for details. The regions to the left and right of the dashed line at $\mu_{r}=1$ correspond to photodarkening and photobleaching photopolymerization, respectively.

to the derivative of $\phi$ at the inflection point overestimating the gradual decay of the conversion profile. The asymmetry of the profile about this point is a contributing factor as well. The estimates of the width given by (5) and (7) for the respective cases of photoinvariance $\left(\mu_{r}=1\right)$ and strong photobleaching $\left(\mu_{r} \gg 1\right)$ are, however, in much better agreement with the widths that are seen directly from the conversion profiles, both of which are plotted in Fig. 4(b). In these two cases, $\phi_{i}$ is much closer to $1 / 2$ and the conversion profiles exhibit a higher degree of symmetry about the inflection point. As a result, the value of $\phi^{\prime}(0)$ gives a better representation of the spatial variation of the conversion fraction near the interface. Thus, we can conclude that our expression for the interfacial width $w$ given by (9) yields accurate quantitative estimates when $\mu_{r} \geqslant 1$ (viz. below the white line in Fig. 3) due to the symmetry of the conversion profile. In the region
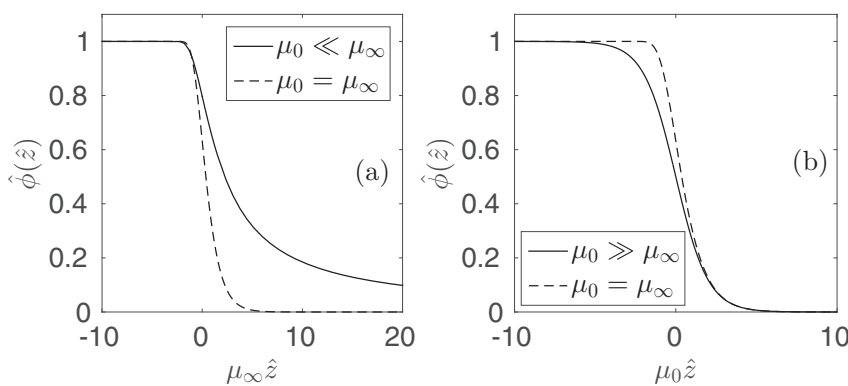

FIG. 4. Characteristic traveling-wave conversion profiles in the case of strong photodarkening (a), strong photobleaching (b), and photoinvariant [(a) and (b)] photopolymerization. The variable on the horizontal axes is a dimensionless measure of the distance from the solid-liquid interface defined by the inflection point of $\hat{\phi}$. above this line, the asymmetry of the conversion profile leads to $w$ underestimating the width of the diffuse layer.

\section{THE ROLE OF ISOTHERMAL MASS DIFFUSION IN FPP}

We next introduce a model that incorporates diffusive mass transport under isothermal conditions. We suppose that the conversion fraction $\phi$ evolves according to a nonlinear reaction-diffusion equation given in dimensional terms as

$$
\frac{\partial \phi}{\partial t}=\frac{\partial}{\partial z}\left(D(\phi) \frac{\partial \phi}{\partial z}\right)+K I_{0}(1-\phi) e^{-\bar{\mu} z},
$$

where $D$ is a diffusivity satisfying $D(0)=D_{0}$. We have assumed that the mean absorption coefficient $\bar{\mu}$ is independent of $\phi$ and, hence, we consider only photoinvariant FPP, which facilitates an analytical study of this model. As shown previously, a photoinvariant model for FPP can, in fact, accurately capture experimental data, at least for sufficiently short times for a range of systems $[4,5,13]$. The analysis that follows does not depend crucially on this simplifying assumption; therefore, the results are expected to carry over to the general case, unless stated otherwise.

The dependence of the diffusivity on the conversion fraction reflects the fact that the mobility of the species and, in particular, of the polymer chains will depend on their molecular weight. To estimate a functional form for the diffusivity, we recall the reptation prediction for the selfdiffusion of entangled polymers in a melt $D \sim M_{w}^{-2}$ [28,29], and larger exponents of up to 2.5 observed in concentrated solutions and gels, where $M_{w}$ is the polymer molecular weight [30]. For the purposes of this "minimally extended" model, it will suffice to consider only the inverse proportionality between $D$ and $M_{w}$ rather than attempting to specify a precise exponent, which is unlikely to be suitable for the heterogeneous systems encountered during photopolymerization. Photopolymerization occurs via chain-growth polymerization, which generates polymer chains of various lengths. Relating the molecular weight of the polymer chains that occupy a given control volume to the local conversion fraction $\phi$ is nontrivial because this variable provides little information about this quantity. Therefore, the molecular weight is assumed to be a monotonically increasing function of the conversion fraction of the form $M_{w} / M_{0}=\left(1-\phi / \phi_{*}\right)^{-b}$, where $b>0$ and $M_{0}$ is the molecular weight of a monomer unit. The vertical asymptote at $\phi=\phi_{*} \leqslant 1$ corresponds to diffusion effectively ceasing as a critical conversion is reached. By combining the expressions for $D$ and $M_{w}$, we can propose an empirical functional form for the diffusivity:

$$
D(\phi)= \begin{cases}D_{0}\left(1-\phi / \phi_{*}\right)^{a}, & \phi<\phi_{*}, \\ 0, & \phi>\phi_{*},\end{cases}
$$

where $a>0$. Given the complexity of the distribution in length of polymer chains, we take the exponent $a$ to be an empirical descriptor of the diffuse nature of the front due to mass transfer. Examples of this function are shown in Fig. 5(a). If $0<a<1$, the decay of the diffusivity with increasing $\phi$ is gradual at first, but rapidly steepens as $\phi_{*}$ is approached. The behavior of the diffusivity reverses when $a>1$; in this case, the initial 

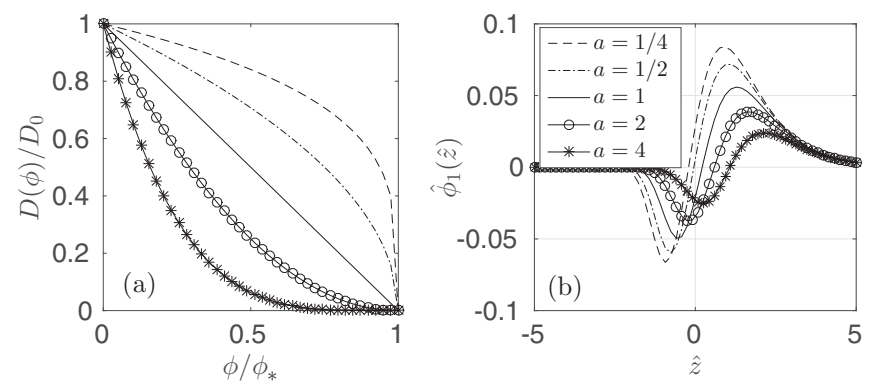

FIG. 5. (a) Sample diffusivities that are used in the extended model with mass transport. These are derived from empirical and phenomenological relationships between the diffusivity, the molecular weight of polymers, and the conversion fraction, and they take the form $D(\phi) / D_{0}=\left(1-\phi / \phi_{*}\right)^{a}$. Here, $D_{0}$ denotes the monomer self-diffusion coefficient; $\phi_{*}$ represents the possibility of a critical density of cross-links at which the network becomes impenetrable to small molecules, causing diffusion to cease; and $a$ is an empirically determined exponent. (b) Corrections to the nondiffusive conversion profile (shown as the dashed line in Fig. 4) in the limit of slow mass diffusion. For a given exponent $a$, the observed conversion profile is a superposition of the nondiffusive profile and the corresponding curve for $\phi_{1}$. Therefore, $\phi_{1}$ indicates how the conversion profile will broaden with time. The legend applies to both panels.

decrease is sharp and the final decay to zero is gradual. To simplify our analysis of this model, we will assume that $\phi_{*}=$ 1. We avoid a formal relationship between $\phi_{*}$ and $\phi_{c}$ at this stage, since the latter criterion corresponds to the formation of a weak gel, which has finite solvent permeability (and is experimentally observed to swell upon prolonged immersion)

In this model, the crossover from isolated chains of polymer diffusing within a bath of monomer to small monomers diffusing into a cross-linked polymer network is captured only by the decrease in the diffusivity with the conversion fraction. Despite its simplicity, the benefit of our model assumption is that analytical progress can be made and used to provide first insights into how mass transport can influence the frontal nature of photopolymerization.

The model is closed by prescribing boundary and initial conditions for the conversion fraction. In this case, we assume the spatial domain is unbounded and given by $z \geqslant 0$. Noflux boundary conditions are imposed at the transparent, illuminated surface, and in the far field; as before, the initial mixture is assumed to be unpolymerized. Thus, we have the following conditions:

$$
\begin{aligned}
\frac{\partial \phi}{\partial z} & =0, \quad z=0, \\
\frac{\partial \phi}{\partial z} & \rightarrow 0, \quad z \rightarrow \infty \\
\phi & =0, \quad t=0 .
\end{aligned}
$$

\section{A. Scaling}

The governing equations are now recast into dimensionless form by choosing suitable scales for the variables in the model. This has the advantage of reducing the number of free parameters in the system, and it aids in identifying the key parameter and time regimes. The time variable $t$ is written in terms of the photoinvariant induction time for an interface defined according to the inflection point, $\tau_{\text {ind }}=\left(K I_{0}\right)^{-1}$. In addition, the spatial coordinate $z$ is written in terms of the approximate width of the interfacial layer $\bar{\mu}^{-1}$. Thus, we set $t=\tau_{\text {ind }} t^{\prime}, z=\bar{\mu}^{-1} z^{\prime}$, and we write $D=D_{0} D^{\prime}$, where primes denote dimensionless quantities. The nondimensional model can be written as (after dropping the primes)

$$
\frac{\partial \phi}{\partial t}=\delta \frac{\partial}{\partial z}\left(D(\phi) \frac{\partial \phi}{\partial z}\right)+(1-\phi) e^{-z}
$$

with a diffusivity given by

$$
D(\phi)=(1-\phi)^{a},
$$

and the following boundary and initial conditions:

$$
\begin{aligned}
\frac{\partial \phi}{\partial z} & =0, \quad z=0, \\
\frac{\partial \phi}{\partial z} & \rightarrow 0, \quad z \rightarrow \infty, \\
\phi & =0, \quad t=0 .
\end{aligned}
$$

The key parameter that controls the dynamics of the model, $\delta$, corresponds to the ratio of the reactive and diffusive time scales:

$$
\delta=\frac{\bar{\mu}^{2} D_{0}}{K I_{0}} .
$$

Thus, $\delta \gg 1$ and $\delta \ll 1$ describe the physical situations in which mass diffusion is much faster and much slower than the effective rate of polymerization, respectively.

\section{B. Limit of slow mass diffusion}

We first focus on the case in which mass diffusion is slow compared to the polymerization reaction, corresponding to $\delta \ll 1$. We find that two distinct time regimes must be considered.

The first of these corresponds to $O(1)$ times. Near the illuminated upper surface, a diffusive boundary layer of width $O\left(\delta^{1 / 2}\right)$ rapidly develops. However, this is not found to significantly influence the behavior of the solution, so we do not discuss it further. Away from the upper surface, i.e., for $z \gg \delta^{1 / 2}$, the diffusive term can be neglected from (14a) and one finds that the conversion fraction settles into the travelingwave profile described by (5), which, in nondimensional form, can be written as

$$
\phi(z, t) \equiv \hat{\phi}_{0}(\hat{z})=1-\exp \left(-e^{-\hat{z}}\right),
$$

where $\hat{z}=z-\log t$.

The second time regime is entered when $t=O\left(\delta^{-1}\right)$. Diffusion becomes important over these long time scales, and it leads to a slow broadening of the interfacial layer. The dynamics in this regime can be studied by first making the change of variable given by $\tau=\delta t$ and $\hat{z}=z-\log t$. The governing equation for the conversion fraction becomes

$$
\frac{\partial \hat{\phi}}{\partial \tau}-\frac{1}{\tau} \frac{\partial \hat{\phi}}{\partial \hat{z}}=\frac{\partial}{\partial \hat{z}}\left(D(\hat{\phi}) \frac{\partial \hat{\phi}}{\partial \hat{z}}\right)+(1-\hat{\phi}) \frac{e^{-\hat{z}}}{\tau} .
$$


Naively taking $\tau \rightarrow 0$ provides an initial condition for this equation, $\hat{\phi}(\hat{z}, \tau \rightarrow 0)=\hat{\phi}_{0}(\hat{z})$; thus, the solution in the second time regime is a continuation of the solution in the first time regime. We look for a solution that is valid for small times, $\tau \ll 1$, by writing

$$
\hat{\phi}(\hat{z})=\hat{\phi}_{0}(\hat{z})+\tau \hat{\phi}_{1}(\hat{z})+O\left(\tau^{2}\right) .
$$

It can be shown that $\hat{\phi}_{1}$ satisfies a linear differential equation given by

$$
\frac{d \hat{\phi}_{1}}{d \hat{z}}-\left(1+e^{-\hat{z}}\right) \hat{\phi}_{1}=-\frac{d}{d \hat{z}}\left(D\left(\hat{\phi}_{0}\right) \frac{d \hat{\phi}_{0}}{d \hat{z}}\right),
$$

with $\hat{\phi}_{1} \rightarrow 0$ as $\hat{z} \rightarrow \infty$. The solution can be written as

$$
\begin{aligned}
\hat{\phi}_{1}(\hat{z})= & \exp \left(\hat{z}-e^{-\hat{z}}\right) \\
& \times \int_{\hat{z}}^{\infty} \exp \left(-s+e^{-s}\right) \frac{d}{d s}\left(D\left(\hat{\phi}_{0}\right) \frac{d \hat{\phi}_{0}}{d s}\right) d s .
\end{aligned}
$$

Using the diffusivity given in (14b), a closed-form expression for $\hat{\phi}_{1}$ can be obtained for any power $a$; in particular,

$$
\hat{\phi}_{1}(\hat{z})=\frac{a^{2}(1+a)+(2+a)\left[a e^{\hat{z}}+e^{2 \hat{z}}-\exp \left(2 \hat{z}+a e^{-\hat{z}}\right)\right]}{a^{3} \exp \left[(1+a) e^{-\hat{z}}+\hat{z}\right]} .
$$

In the limit of constant diffusivity, $a \rightarrow 0$, this expression reduces to

$$
\hat{\phi}_{1}(\hat{z})=\frac{1}{6}\left(3 e^{\hat{z}}-2\right) \exp \left(-2 \hat{z}-e^{-\hat{z}}\right) .
$$

By plotting $\hat{\phi}_{1}$ as a function of $\hat{z}$ for different values of $a$, we can examine how the conversion fraction is expected to deviate from the nondiffusive conversion profile given by (16) and, in particular, how the interfacial layer begins to broaden. Such curves are shown in Fig. 5(b). We first notice that $\hat{\phi}_{1}(\hat{z}=0) \neq 0$, corresponding to a shift in the inflection point and the location of the interface. Secondly, we see that these functions are not symmetric about $\hat{z}=0$. The ramification of this is that the interfacial layer does not broaden in a symmetric manner, but rather there is more spreading ahead of the front than behind it. A possible mechanism for asymmetric broadening is the variation of the diffusivity across the interfacial layer; however, the asymmetry is still present when the diffusivity is constant, corresponding to $a=0$, implying it must originate from elsewhere. Indeed, we find that the asymmetry is inherited from that in the nondiffusive profile; the nonconstant diffusivity across the interface merely magnifies this.

The influence of slow mass diffusion on the position and width of the interfacial layer over intermediate times satisfying $t \ll \delta^{-1}$ can be determined by analyzing the approximate solution for the conversion fraction given in (18). It can be shown that these two quantities are given by

$$
z_{f}(t) \simeq \log t-\delta t\left[e^{1} \hat{\phi}_{1}^{\prime \prime}(0)\right], \quad t \ll \delta^{-1}
$$

and

$$
w(t) \simeq e^{1}\left[1+\delta t e^{1} \hat{\phi}_{1}^{\prime}(0)\right], \quad t \ll \delta^{-1},
$$

respectively. Thus, mass diffusion leads to a small linear drift in the position of the interface, which itself is caused by the asymmetric broadening of the interfacial layer. If the broadening was symmetric in the sense that $\hat{\phi}_{1}^{\prime \prime}(0)=0$, corresponding to the case in which the inflection points of $\hat{\phi}_{0}$ and $\hat{\phi}_{1}$ coincide with each other, then mass diffusion would be a higher-order effect and the original expression for the position of the interface in the limit of no mass transport, $z_{f}(t)=\log t$, would be recovered. The approximation for $w$ given in (24) indicates that mass diffusion leads to a linear broadening of the interfacial layer over intermediate times.

To explore the dynamics that occur over larger times, the boundary-value problem (14) is solved numerically for specific values of $\delta$ and $a$. Changes in $a$ and $\delta$ only affect the qualitative structure of the solutions, so we shall only report the results that are obtained for one set of parameter values. The results from a simulation with $\delta=10^{-2}$ and $a=2$ are shown in Fig. 6. Panel (a) displays the evolution of the conversion fraction in a frame that travels with the interface. A significant broadening of the interfacial layer occurs, particularly ahead of the front where the (oligomeric and monomeric) species are highly mobile. The mobility decreases sharply behind the front due to the nonconstant diffusivity, effectively freezing the morphology of the profile in this region. Also shown in this panel is the two-term approximation of the conversion profile for intermediate times given by (18), which is in excellent agreement with the full numerical solution. Figures 6(b) and 6(c) show how the position of the interface and the width of the
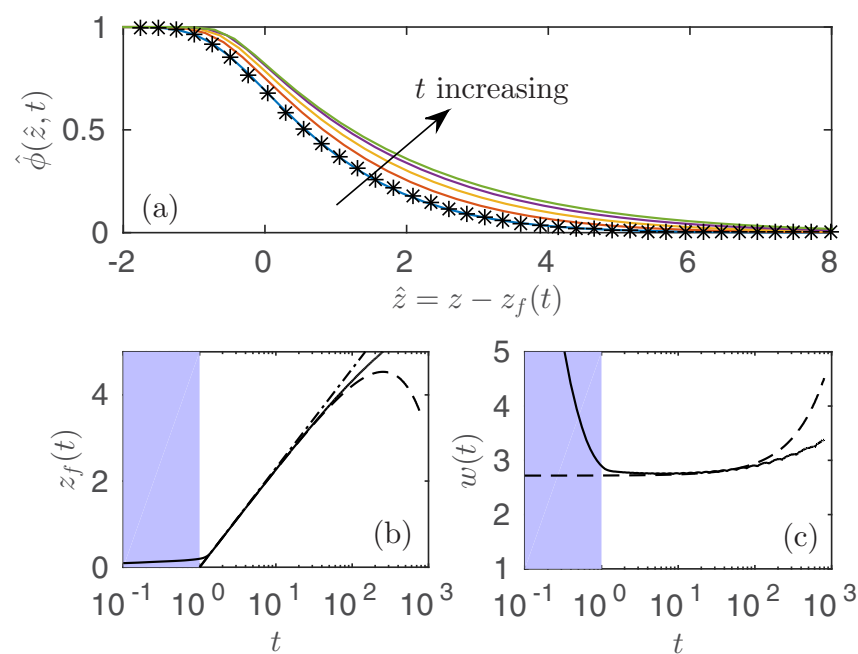

FIG. 6. (Color online) FPP kinetics under isothermal conditions when mass diffusion is slow relative to the polymerization reaction, as characterized by $\delta \ll 1$. Snapshots of the conversion profile at (nondimensional) times 80, 240, 400, 560, and 720 (a). Due to mass transport, the interfacial profile broadens with time. The symbols denote an asymptotic solution given by (16), (18), and (21). Evolution of the location (b) and width (c) of the interface. The highlighted regions in light blue correspond to an initial time regime whereby the interfacial layer has yet to develop fully; thus, neither the inflection point nor the associated width are physically meaningful here. The dash-dotted line in panel (b) is the approximation of the interface position in the limit of no mass transport, $z_{f}(t)=\log t$. The dashed lines in panels (b) and (c) are approximations given by (23) and (24) that account for mass transport. Parameter values are $\delta=10^{-2}$ and $a=2$. 
interfacial layer evolve in time, respectively. For small times given by $t<1$, corresponding to the light-blue regions of these two panels, the inflection point lies within the diffusive boundary layer near the transparent upper surface, and the conversion profile has yet to fully develop an interfacial layer. Therefore, neither the inflection point nor the corresponding width are physically meaningful for $t<1$, so we do not consider this time interval further. For larger times given by $t>1$, Fig. 6(b) shows how the position of the interface slowly deviates from the logarithmic behavior observed when mass transport is negligible (shown as the dash-dotted line). The approximation of the interface position (23), shown as the dashed line, is in good agreement with the numerical solution for the times in which it is valid, $t \ll \delta^{-1}$. Figure 6(c) shows that for $O(1)$ times, the width of the diffuse interface remains approximately constant and equal to the value of $e^{1}$ predicted from the model without mass transport. For longer times satisfying $t \ll \delta^{-1}$, the width increases linearly in accordance with the approximation given by (24), which is indicated by the dashed line in this panel. This linear growth slows down when $t>\delta^{-1}$, indicating a transition into a time regime with diffusion-dominated dynamics. In such a regime, the width is expected to scale like $w \sim t^{1 / 2}$. Further analysis of the numerically computed width in Fig. 6(c) reveals that the $t^{1 / 2}$ behavior is indeed approached for sufficiently long times. By comparing the widths predicted by $w$ to those seen visually from the conversion fractions, we find that on very long time scales, $w$ underestimates the widths due to asymmetric broadening of the conversion profiles and their slow decay to zero from the inflection point.

\section{Limit of moderate and fast diffusion}

When mass diffusion occurs on a time scale that is similar to or faster than that of the photopolymerization reaction, i.e., when $\delta=O(1)$ or larger, a significant broadening of the conversion profile occurs even before the surface of the mixture is fully converted, corresponding to complete network formation. Such behavior can be seen in Fig. 7, which shows the results of two simulations of (14) when $\delta=1$ and for two values of the exponent $a$ that appears in (14b). The strong broadening due to rapid mass transport essentially destroys the frontal nature of FPP.

\section{Mean extent of polymerization}

To further characterize how mass diffusion influences the dynamics of FPP, it is instructive to examine the evolution of the mean conversion fraction. This is defined as

$$
\langle\phi\rangle_{\delta}(t)=\frac{1}{H} \int_{0}^{H} \phi(z, t ; \delta) d z,
$$

where the spatial domain has been truncated at $z=H$ in order to obtain meaningful results. This quantity estimates, on average, the mean extent of polymerization throughout the mixture. In the limit of slow diffusion, $\delta \rightarrow 0$, the solution for $\phi$ is given by (16), and, therefore, this integral can be evaluated to give

$$
\langle\phi\rangle_{0}(t)=1-\frac{1}{H}\left[E_{1}\left(t e^{-H}\right)-E_{1}(t)\right],
$$
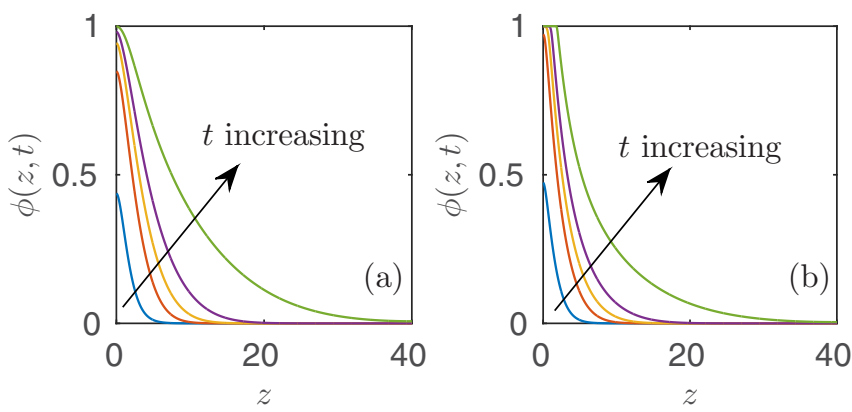

FIG. 7. (Color online) Conversion profiles at various times when mass transport occurs on a similar time scale to the polymerization reaction. The two panels distinguish results obtained using different exponents in the diffusivity (14b). We take $a=2 / 3$ (a) and $a=2$ (b), corresponding to diffusivities with qualitatively different behaviors; see Fig. 5. In both panels, $\delta=1$. The profiles are shown at (nondimensional) times $t=1,5,10,20$, and 100. They indicate that significant broadening occurs in this regime, destroying the frontal nature of FPP. Moreover, variations in the exponent $a$ only result in small changes to the conversion profiles.

where $E_{1}$ is an exponential integral defined as

$$
E_{1}(\xi)=\int_{1}^{\infty} \frac{e^{-\xi s}}{s} d s .
$$

In the limit of fast mass diffusion, $\delta \rightarrow \infty$, the conversion profiles are spatially uniform, $\phi(z, t)=\phi(t)$; thus, a differential equation for the mean conversion fraction can be obtained by integrating (14a) with respect to $z$ and applying the no-flux boundary conditions in (14c) and (14d). Upon solving this equation using the initial condition $\langle\phi\rangle_{\infty}(0)=0$, we find that

$$
\langle\phi\rangle_{\infty}(t)=1-\exp \left[-H^{-1} t\left(1-e^{-H}\right)\right] .
$$

The fact that $\langle\phi\rangle_{0} \neq\langle\phi\rangle_{\infty}$ for each value of $t>0$ suggests that mass diffusion not only affects the spatial distribution of polymers, but also the overall extent of monomer conversion at any given time. This result is surprising, as it suggests that mass diffusion can effectively increase conversion, for a fixed photon dose. Intuitively, this can be rationalized in terms of a positive feedback imposed on the governing equation for $\phi$ [cf. (1a) for simplicity] due to diffusion flattening the conversion profile and locally reducing the value $\phi$, thus increasing $(1-\phi)$ and the rate of reaction. Figure 8 illustrates both of these points in greater detail. In particular, panel (a) shows, for a fixed time $t$, how larger values of $\delta$, corresponding to faster diffusion, lead to a redistribution of the polymer-rich phase across the domain. Panel (b) shows the evolution of the mean conversion fraction for different values of $\delta$. From this panel, it can be seen that faster mass diffusion is responsible for increasing the net amount of polymer that is produced in a given amount of time. This is a consequence of diffusion replenishing the monomer near the transparent substrate where the intensity of radiation is the highest, both of which are factors that allow for an acceleration of the local conversion into polymer.

Interestingly, these results differ from those of Terrones and Pearlstein [25], who show that in a perfectly photobleaching system, the mean conversion fraction is independent of the 

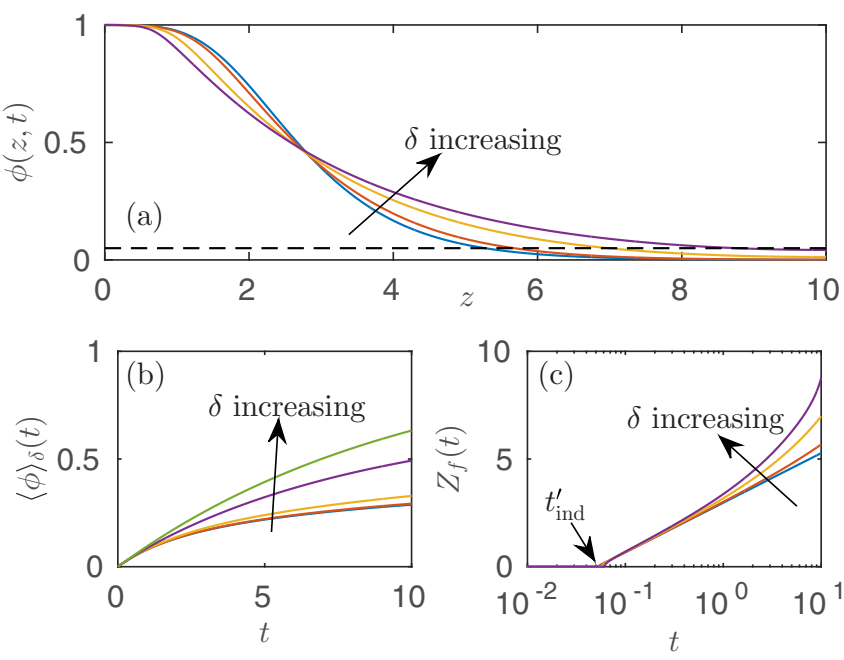

FIG. 8. (Color online) (a) Increasing the nondimensional diffusivity $\delta$, corresponding to faster mass diffusion, redistributes the polymer in such a way that, for a fixed (nondimensional) time $t=10$, it is spread further across the spatial domain. The dashed line denotes a critical conversion fraction, $\phi_{c}=0.05$, that can be used as an alternative definition of the solid-liquid interface. (b) Diffusion leads to a replenishment of the monomer near the transparent substrate located at $z=0$, and this, in turn, increases the net production of polymer, which can be measured through the mean conversion fraction $\langle\phi\rangle_{\delta}$, defined in (25). (c) Front propagation kinetics, defined using the criteria $\phi\left(Z_{f}(t), t\right)=\phi_{c}=0.05$, shows that FPP is accelerated due to mass diffusion. The nondimensional induction times are given approximately by $t_{\text {ind }}^{\prime} \simeq \log \left[1 /\left(1-\phi_{c}\right)\right]$. The corresponding profiles at $t=10$ are shown in panel (a). In panels (a) and (c), the values of $\delta$ are $0,0.1,0.5$, and 1 , whereas in panels (b) they are $0,0.1,1,10$, and $\delta \rightarrow \infty$. In all cases, $a=2$ with $H=10$.

nondimensional diffusivity $\delta$. In this case, the increase in the rate of polymerization that occurs due to the replenishment of the monomer near the transparent surface is exactly offset by the decrease in radiation intensity throughout the mixture due to this buildup of strongly absorbing molecules. We emphasize that such behavior is specific to systems that exhibit perfect photobleaching, and it would also be observed in our model if it considered this type of photopolymerization.

\section{E. An alternative definition of the interface}

In the absence of mass diffusion, it is possible to define the position of the interface in several equivalent ways, for instance using the inflection point of the conversion profile or a critical value of the conversion fraction. However, in the presence of mass diffusion, the lack of a time-invariant traveling-wave conversion profile means that different dynamics can be observed depending on the choice of interface definition.

In FPP fabrication, the front position is naturally defined by the selective dissolution of the liquid phase, yielding a sharp solid boundary $[4,5,13]$. Effectively, in terms of our model, this corresponds to defining a (system-dependent) critical value of the conversion fraction $\phi_{c}$, rather than the inflection point. This criterion has the advantage of being unambiguous, and it is found to be robust to a variety of selective solvents [4]: any material below the measured $\phi_{c}$ is washed off.
Vitale et al.[13] have recently succeeding in separately measuring full conversion profiles and front kinetics based on $\phi_{c}$, obtaining remarkably good agreement between those independent measurements. From a fabrication point of view, it is thus preferable to compute front kinetics using this empirical $\phi_{c}$ criterion, instead of the profile's inflection point. To explore how the dynamics change upon using $\phi_{c}$ to define the interface, we introduce the variable $Z_{f}$ for the new front position, defined implicitly through the relation $\phi\left(Z_{f}(t), t\right)=\phi_{c}$. Figure $8(\mathrm{c})$ shows the characteristic evolution of $Z_{f}$ for values of $\delta$ satisfying $0 \leqslant \delta \leqslant 1$ when $\phi_{c}=0.05$. The corresponding final conversion profiles are shown along with $\phi_{c}$ in Fig. 8(a). According to this description of the interface, mass diffusion serves to accelerate the motion of the front by essentially pushing regions of $\phi_{c}$ further into the unpolymerized mixture. This is in stark contrast to when the interface was defined in terms of the inflection point, where a slowing of the front was observed; see, for example, Fig. 6. However, if a larger value of $\phi_{c}$ was used, particularly one that satisfies $\phi_{c}>0.47$, then a similar deceleration would be observed due to diffusion pushing these regions of $\phi_{c}$ in the upbeam direction, that is, toward the transparent substrate. From this we can conclude that particular care must be taken when choosing a suitable definition for the interface when mass transport is non-negligible.

For the moderate values of $\delta$ used in Fig. 8(c), the induction times are given approximately by the nondimensionalized version of (6), namely $t_{\text {ind }}^{\prime} \equiv t_{\text {ind }} / \tau_{\text {ind }} \simeq \log \left[1 /\left(1-\phi_{c}\right)\right]$. For larger values of $\delta$, this approximation breaks down and there is a marked increase in the induction time $t_{\text {ind }}^{\prime}$, although the broadening of the interfacial layer is so severe in this regime that FPP does not occur.

\section{MODEL VALIDATION WITH FPP EXPERIMENTS: OPTICAL ATTENUATION AND MASS DIFFUSION EFFECTS}

We have performed photopolymerization experiments to evaluate how the optical attenuation coefficients and mass transport affect the profile shape and the sharpness of the polymerization front. Our experimental configuration mimics that shown in Fig. 1(a); a monomer-rich bath is placed on a thermally cured PDMS substrate and covered with a glass surface. Collimated light from a monochromatic (365 nm) uv source is passed through a photomask placed on top of the glass surface to selectively illuminate a well-defined region of the bath, away from the lateral container boundaries. A 100 W mercury flood lamp (Spectroline SB-100P) was used as the light source. The intensity of radiation entering the bath was measured with a UVItec RS-365 digital radiometer. Following light exposure, the specimen was developed by selective dissolution, with ethanol and acetone, and cross-sectioned in order to quantify the conversion profile along the $z$ direction (or depth).

A Bruker Tensor 27 spectrometer and a Hyperion microscope were employed to compute polymerization conversion of the samples by Fourier transform infrared spectroscopy (FTIR), enabling direct monitoring of the spatiotemporal evolution of the conversion fraction $\phi$ during FPP. In particular, the conversion profile was monitored by analyzing the variation 


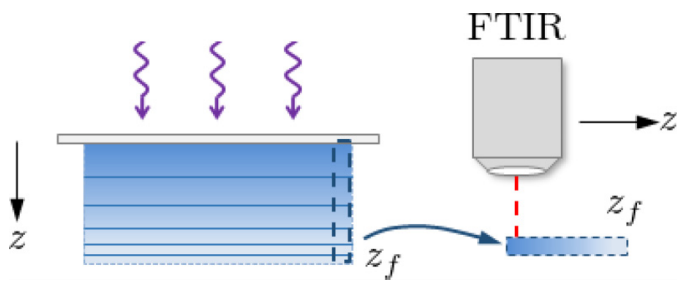

(a)
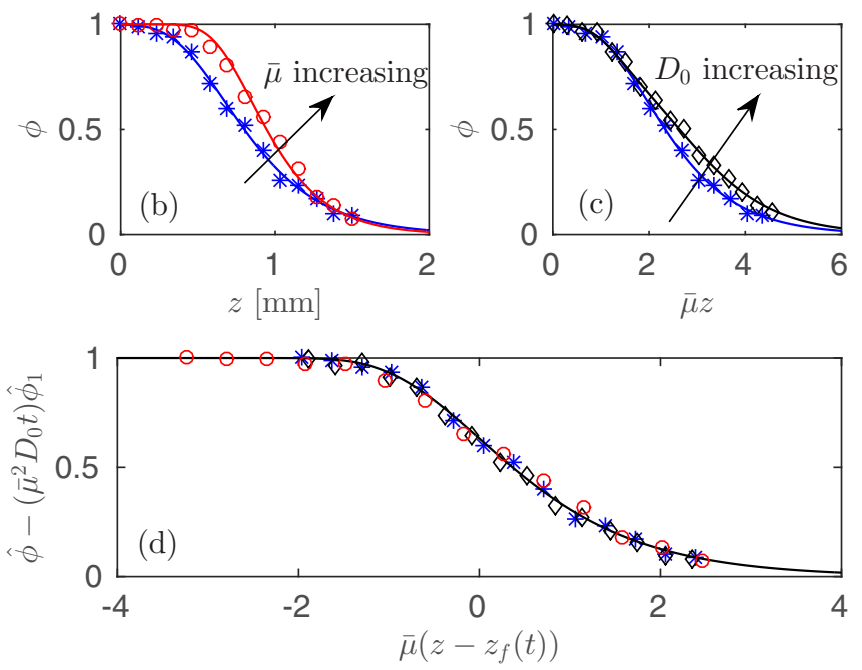

FIG. 9. (Color online) (a) Fourier transform infrared (FTIR) spectroscopy is employed to directly measure the conversion profiles $\phi$ at selected times. The polymerized specimens are developed and fractured into thin slices along the vertical $z$ direction. The spectra of these samples are then measured along the $z$ direction using FTIR microscopy. For additional details, see Vitale et al. [13]. (b)-(d) Experimental (symbols) and theoretical (lines) conversion profiles obtained from monomer-rich baths with different optical attenuation coefficients but limited mass transport (b), and similar absorption coefficients but different degrees of mass transport (c). All data can be collapsed onto a master curve (d) by an appropriate rescaling of the spatial variable and by subtracting off the diffusive contribution to the profile. Blue stars, red circles, and black diamonds correspond to bath formulations that are based on photoresist NOA81, NOA81 with absorbing dye, and NOA81 with an additional low-molecular-weight acrylic monomer, respectively; see text for further details.

of the thiol reactive functionality absorption band; for further details, see Vitale et al. [13]. A schematic of this process is shown in Fig. 9(a).

In all experiments reported, the monomer-rich bath is a commercial Norland NOA81 thiol-ene photoresist or custommade formulations based on NOA81. The base photoresist NOA81 has been shown to exhibit frontal kinetics compatible with photoinvariant FPP at relatively early times. Further, this system behaves regularly over a wide temperature range, exhibiting limited mass transport [4,5], negligible shrinkage upon polymerization, and small temperature rises during experiments [13]. These last two points suggest that the density of the mixture remains approximately constant during the solidification process, thus inhibiting the onset of buoyancydriven convection. This mode of convective transport can have important consequences for frontal processes: the flow of the denser phase away from the polymerization front may broaden the interfacial layer or lead to the formation of fingers via a Taylor instability, which, in turn, will drive the breakup of the planar front $[31,32]$.

We first examine how varying the optical attenuation coefficient of the base NOA81 resist leads to changes in the characteristics of the polymerization front. Toward that end, we have formulated a second resist by incorporating a lightabsorbing dye (Cresol Red, Sigma-Aldrich, 0.5 wt. \%) to the original, thereby increasing the optical attention coefficient, $\bar{\mu}$, from 2.92 to $3.81 \mathrm{~mm}^{-1}$, a rise of about $30 \%$. The two corresponding conversion fractions are plotted as functions of the (dimensional) vertical coordinate $z$ and shown as symbols in Fig. 9(b). A visual inspection of the experimental data shows that the width of the interfacial layer decreases in the formulation with the larger absorption coefficient. The data can be well fitted to the solution of the photoinvariant FPP model without mass transport given in (5) by taking $K I_{0} t$ as the single fitting parameter. We note that the attenuation coefficients are independently measured, prior to exposure, via a simple transmission reading of specimens of known thickness and via the Beer-Lambert law. Further, both $t$ and $I_{0}$ are experimentally known so that the constant $K$ is thus the only undetermined parameter that must be fit to the data. The fitted solutions are shown as the smooth curves, and the agreement with the experimental data is excellent.

According to the analysis of Sec. II, the experimental data should collapse onto a master curve given by $\hat{\phi}=1-$ $\exp \left(-e^{-\hat{z}}\right)$ once it is written in terms of the nondimensional traveling-wave coordinate $\hat{z}=\bar{\mu}\left[z-z_{f}(t)\right]$, where $z_{f}$ is the dimensional location of the inflection point given in (5). Figure 9(d) shows that this is indeed the case, and all data fit tightly on this master profile. Thus, the two conversion profiles shown in panel (b) of this figure are essentially just stretched versions of the same master curve, with the stretch, and hence the width of the interfacial layer, being controlled by the magnitude of the optical attenuation coefficient.

To explore how mass transport affects the polymerization front, we carry out experiments using a modified NOA81 formulation that has an additional monomer of low molecular weight, namely hexanediol diacrylate (Sigma-Aldrich, 10 wt. \%). While this modification increases the overall mobility of the components in the mixture, it also leads to a decrease in the absorption coefficient from 2.92 to $2.64 \mathrm{~mm}^{-1}$. To account for variations in the conversion profiles due to different absorption coefficients and, therefore, to isolate the broadening of the interfacial layer due to mass diffusion, the experimental data have been plotted in Fig. 9(c) as a function of a nondimensionalized coordinate $\bar{\mu} z$. Evidently, when the diffusivity is increased, there is a significant broadening of the conversion profile, mainly ahead of the polymerization front, which agrees qualitatively with our theoretical predictions.

We note that by adding an acrylic monomer, polymerization proceeds by two simultaneous mechanisms: free-radical chain growth of the acrylates, both within the matrix and with the acrylate monomer itself, in addition to the thiol-ene step growth. The results presented in Fig. 9 concern only the conversion of the thiol functionality. Further, we have measured the front propagation rate of the neat and doped systems, and we concluded that these remain identical when 
the change in optical attenuation coefficient is accounted for. We can therefore conclude that the change in profile shapes due to the addition of the low-molecular-weight monomer is caused by an increase in the diffusion coefficient.

To make a quantitative comparison between theory and experiment, we first notice that the close resemblance of the two conversion profiles suggests that diffusion occurs on a slower time scale than the photopolymerisation reaction, corresponding to the regime where the nondimensional number $\delta$, defined in (15), is small. We therefore attempt to fit the approximate solution given by (16), (18), and (21) to the experimental data associated with the modified formulation (shown as the black diamonds), using $\delta$, the nondimensional time $t^{\prime}$, and $a$ as fitting parameters. In terms of physical quantities, fitting $t^{\prime}$ and $\delta$ provides values for the effective rate constant $K$ and the diffusivity $D_{0}$, respectively. We find that $\delta \simeq 0.10$ and $t^{\prime} \simeq 8.8$ so that $\delta \ll 1$ and $\delta t^{\prime}<1$, which are requirements for this approximate solution to be valid. In addition, we find that $a \simeq 0.67$. As shown in Fig. 9(c), the match between the fitted solution and the experimental data is exceptional, thus indicating that the extended model with mass transport is able to accurately capture the evolution of the interfacial layer and the conversion profile. Finally, we note that the experimental data associated with the modified formulation can further be collapsed onto the master curve shown in Fig. 9(d). This is achieved by first subtracting off the diffusive contribution to the profile given in dimensional terms as $\bar{\mu}^{2} D_{0} t \hat{\phi}_{1}$, with $\hat{\phi}_{1}$ given by (21), and then writing the profile in terms of the nondimensional traveling-wave coordinate $\bar{\mu}\left[z-z_{f}(t)\right]$, with $z_{f}$ given by the expression in (5). In general, such a subtraction can be performed provided that the dimensionless quantity $\bar{\mu}^{2} D_{0} t \ll 1$. After carrying out this procedure, we see from Fig. 9(d) that the experimental data collapse remarkably well onto the master profile, thus highlighting the underlying FPP dynamics occurring in the presence of significant mass transport.

\section{DISCUSSION AND CONCLUSION}

In this paper, two mathematical models of photopolymerization, each considering different physical effects, have been studied. Particular attention has been paid to determining when photopolymerization can be considered "frontal" in nature, and, when this is the case, understanding the relationship between the physical parameters of the system and the width of the interfacial layer separating solid-rich from liquid-rich phases. The results from this study indicate that photopolymerization will be frontal provided that mass transport is sufficiently slow, as measured relative to the rate of the photopolymerization reaction, and the optical attenuation is strong. In general, the optical attenuation coefficients play a primary role in selecting the width of the interfacial layer, with the width decreasing as these coefficients increase. We also find that mass diffusion, which can be effectively controlled by the mass of the photoresist monomers, or through the addition of low-molecular-weight monomers, can enhance the overall rate of photopolymerization and accelerate the propagation of FPP waves.

From a practical standpoint, a simple way to tailor the conversion profiles that are achieved via FPP is by varying the optical attenuation coefficients. As demonstrated by Cabral and Douglas [5], these coefficients can be modulated by the addition of nanoparticles to the initial liquid monomer bath. In fact, it was shown that the mean optical attenuation coefficient could be increased by as much as one order of magnitude while still maintaining qualitatively similar FPP dynamics. Although the conversion profiles were not measured [5], the minimal model of Sec. II gives the prediction that the width of the interfacial layer would decrease by a factor of 10 , a notable sharpening indeed. However, two main issues may be important when using this approach to modify conversion profiles. First, embedding nanoparticles could significantly alter the thermal properties of the mixture, leading to enhanced heat generation and conduction. If thermal conduction cannot compensate for increased heat generation, large temperature rises could initiate mass-transport phenomena that would in turn broaden the front. Second, in the absence of thermal effects, there is a limit on how thin the interfacial region can be made through adjustments in the optical attenuation coefficient before mass diffusion becomes relevant. The analysis of Sec. III indicates that the relevance of mass diffusion is determined through the nondimensional parameter $\delta=\bar{\mu}^{2} D_{0} /\left(K I_{0}\right)$, which is proportional to the square of the attenuation coefficient. Thus, mass diffusion effectively becomes much stronger as the mean attenuation coefficient increases, possibly counterbalancing the sharpening of the front that would otherwise occur.

The excellent comparisons between theory and experiment demonstrate that simple models of photopolymerization can accurately capture the behavior of physically relevant observable quantities. Moreover, the predictive power of such models, in combination with the novel insights that are obtained from studying them, can be used to tailor photopolymerization experiments, thus enabling the fabrication of innovative network solids with desirable, modulated physical characteristics.

\section{ACKNOWLEDGMENTS}

We thank The Engineering and Physical Sciences Research Council (EPSRC) for a Manufacturing with Light grant EP/L022176/1, and Jack. F. Douglas (NIST) for stimulating discussions.
[1] C. Decker, Prog. Polym. Sci. 21, 593 (1996).

[2] C. Decker, Macromol. Rapid Commun. 23, 1067 (2002).

[3] J.-P. Fouassier and J. F. Rabek, Radiation Curing in Polymer Science and Technology: Practical Aspects and Applications (Elsevier, Amsterdam, 1993), Vol. 4.
[4] J. T. Cabral, S. D. Hudson, C. Harrison, and J. F. Douglas, Langmuir 20, 10020 (2004).

[5] J. T. Cabral and J. F. Douglas, Polymer 46, 4230 (2005).

[6] J. A. Warren, J. T. Cabral, and J. F. Douglas, Phys. Rev. E 72, 021801 (2005). 
[7] J. A. Pojman, in Polymer Science: A Comprehensive Reference, edited by K. Matyjaszewski and K. M. Möller (Elsevier, Amsterdam, 2012), pp. 957-980.

[8] C. Harrison, J. T. Cabral, C. M. Stafford, A. Karim, and E. J. Amis, J. Micromech. Microeng. 14, 153 (2004).

[9] M. Sangermano, G. Malucelli, E. Amerio, A. Priola, E. Billi, and G. Rizza, Prog. Org. Coat. 54, 134 (2005).

[10] G. B. dos Santos, R. V. Monte Alto, H. R. Sampaio Filho, E. M. da Silva, and C. E. Fellows, Dent. Mater. 24, 571 (2008).

[11] J. Elisseeff, K. Anseth, D. Sims, W. McIntosh, M. Randolph, M. Yaremchuk, and R. Langer, Plast. Reconstr. Surg. 104, 1014 (1999).

[12] Z. T. Cygan, J. T. Cabral, K. L. Beers, and E. J. Amis, Langmuir 21, 3629 (2005).

[13] A. Vitale, M. G. Hennessy, O. K. Matar, and J. T. Cabral, Macromolecules 48, 198 (2015).

[14] V. V. Ivanov and C. Decker, Polym. Int. 50, 113 (2001).

[15] A. K. O'Brien and C. N. Bowman, Macromolecules 36, 7777 (2003).

[16] C. N. Bowman and C. J. Kloxin, AIChE J. 54, 2775 (2008).

[17] G. Terrones and A. J. Pearlstein, Macromolecules 34, 8894 (2001).

[18] G. Terrones and A. J. Pearlstein, Macromolecules 34, 3195 (2001).
[19] N. B. Cramer, T. Davies, A. K. O'Brien, and C. N. Bowman, Macromolecules 36, 4631 (2003).

[20] M. D. Goodner and C. N. Bowman, Chem. Eng. Sci. 57, 887 (2002).

[21] S. K. Reddy, N. B. Cramer, and C. N. Bowman, Macromolecules 39, 3673 (2006).

[22] S. K. Reddy, N. B. Cramer, and C. N. Bowman, Macromolecules 39, 3681 (2006).

[23] O. Okay and C. Bowman, Macromol. Theor. Simul. 14, 267 (2005).

[24] G. Terrones and A. J. Pearlstein, Macromolecules 36, 6346 (2003).

[25] G. Terrones and A. J. Pearlstein, Macromolecules 37, 1565 (2004).

[26] S. Bayou, M. Mouzali, F. Aloui, L. Lecamp, and P. Lebaudy, Polym. J. 45, 863 (2013).

[27] H. Li, Y. Qi, and J. T. Sheridan, J. Opt. Soc. Am. B 31, 2648 (2014).

[28] P. G. de Gennes, J. Chem. Phys. 55, 572 (1971).

[29] M. Doi and S. F. Edwards, The Theory of Polymer Dynamics (Clarendon, Oxford, 1988).

[30] T. P. Lodge, Phys. Rev. Lett. 83, 3218 (1999).

[31] J. A. Pojman, R. Craven, A. Khan, and W. West, J. Phys. Chem. 96, 7466 (1992).

[32] J. A. Pojman, V. M. Ilyashenko, and A. M. Khan, J. Chem. Soc., Faraday Trans. 92, 2825 (1996). 